\title{
Equivalence of phase-oscillator and integrate-and-fire models
}

\author{
Antonio Politi ${ }^{1, *}$ and Michael Rosenblum ${ }^{2, \dagger}$ \\ ${ }^{1}$ Institute for Complex Systems and Mathematical Biology and SUPA, University of Aberdeen, Aberdeen AB24 3UE, United Kingdom \\ ${ }^{2}$ Department of Physics and Astronomy, University of Potsdam, Karl-Libknecht-Strasse 24/25, 14476 Potsdam, Germany
}

(Received 4 December 2014; revised manuscript received 5 March 2015; published 29 April 2015)

\begin{abstract}
A quantitative comparison of various classes of oscillators (integrate-and-fire, Winfree, and Kuramoto-Daido type) is performed in the weak-coupling limit for a fully connected network of identical units. An almost perfect agreement is found, with only tiny differences among the models. We also show that the regime of self-consistent partial synchronization is rather general and can be observed for arbitrarily small coupling strength in any model class. As a byproduct of our study, we are able to show that an integrate-and-fire model with a generic pulse shape can be always transformed into a similar model with $\delta$ pulses and a suitable phase response curve.
\end{abstract}

DOI: 10.1103/PhysRevE.91.042916

PACS number(s): 05.45.Jn

\section{INTRODUCTION}

Many studies of neural networks and, generally, of coupled oscillators are based on the assumption that the relevant dynamical properties can be reproduced by restricting the study to dynamical systems characterized by a single variable: the phase. In spite of its simplicity, this setup is indeed able to produce a wealth of nontrivial phenomena, ranging from the synchronization transition [1-3], to self-consistent partial synchronization [4-6], and including chimera states [7,8], to name just a few.

The first such model was proposed by Winfree in 1967 to characterize biological rhythms $[9,10]$. In the weak-coupling limit, it may reduce to the famous Kuramoto model $[1,2,11]$, which is currently much used to investigate the synchronization properties of various setups. While in the Winfree model the coupling depends on the absolute value of the oscillator phases, in the Kuramoto model it depends sinusoidally on phase differences. In fact, the Kuramoto model has been generalized to the so-called Kuramoto-Daido model [12], where the coupling is a generic function of the phase difference.

Independently, yet another class of oscillators is being investigated: the so-called pulse-coupled integrate-and-fire oscillators. Here, a single phaselike variable, describing the membrane potential, increases linearly until it reaches a threshold, is thereby reset to some specific value, and simultaneously triggers the emission of a pulse that is responsible for the mutual coupling. The effect of the pulse onto the receiving oscillator is quantified by the phase response curve. The simplest of such models was proposed in the context of heart activity [13], but is nowadays quite popular in computational neuroscience, where it is widely used to clarify the collective dynamics of neural circuits [14]. A similar and much used model is the leaky integrate-and-fire (LIF) neuron, introduced by Lapicque in 1907, even before physiological mechanisms of pulse transmission were understood [15]. There, the membrane potential evolves exponentially rather than linearly in time.

Nowadays, whenever oscillatory phenomena have to be investigated, integrate-and-fire and Kuramoto-like models are

\footnotetext{
*a.politi@abdn.ac.uk

${ }^{\dagger}$ mros@uni-potsdam.de
}

the most used setups, but it is not clear to what extent the resulting phenomenology is typical of the selected model. A prominent example to illustrate the lack of a general framework is self-consistent partial synchronization (SCPS), a regime where identical oscillators are neither locked, nor completely asynchronous. Kuramoto [4] found evidence of SCPS in a network of identical LIF oscillators in the presence of noise and delayed $\delta$ pulses. Later, van Vreeswijk observed and analyzed this regime in an ensemble of LIF oscillators coupled through smooth pulses and in the absence of external noise [5]. SCPS may also arise in the simple Kuramoto-Sakaguchi model [11] (sine coupling with a phase shift) but only for a particular value of the phase shift, when it is marginally stable. The onset of a robust SCPS regime is, however, possible in a Kuramoto-Sakaguchi-like setup, under the condition that the phase-shift parameter of the sine function depends on the order parameter and the coupling strength [16]. This model can be obtained as a phase approximation of nonlinearly coupled Stuart-Landau oscillators.

Another example of differences among the various setups is emergence of the irregular collective dynamics in an ensemble of heterogeneous LIFs with delayed $\delta$ pulses [17]. The setup is superficially analogous to the Kuramoto ensemble, but chaotic collective oscillations are not possible in the latter model $[18,19]$.

In this paper we compare the various model classes in the minimal setup of identical globally coupled oscillators. In order to carry on a meaningful quantitative analysis, three models (A, B, and C) are selected as follows. Model $A$ is the ensemble of LIF neurons extensively studied in Ref. [20]. By then following Ref. [21], model A is mapped, in the weak-coupling limit, onto a Winfree-type ensemble of oscillators, yielding model B. Finally, model C is obtained as an approximate reduction of model B to a Kuramoto-Daido ensemble.

Our studies reveal that the scenario emerging from the three models is substantially equivalent with a couple of quantitative discrepancies which concern the fully synchronous regime: (i) the dependence of the period on the coupling strength is different in model A already at the leading order; (ii) its stability differs in model C. Finally, the equivalence between models $\mathrm{A}, \mathrm{B}$, and $\mathrm{C}$ implies that a generic LIF model with pulses of finite width can be mapped onto a model of pulsecoupled oscillators and $\delta$-like pulses, which can be more easily 
simulated with event-driven algorithms. To test this conjecture a model of the latter type is introduced (model D).

More specifically, in Sec. II, we introduce the various model classes, discuss their mutual relationships, and briefly recall the most common asymptotic regimes. Section III is devoted to a quantitative comparison of the models $\mathrm{A}, \mathrm{B}, \mathrm{C}$, and D: in practice the analytically estimated stability spectra of the splay and synchronous states, as well as the numerically obtained features of the SCPS are mutually compared. Section IV is devoted to a perturbative analysis of SCPS in the KuramotoDaido setup. The resulting frequency of SCPS are found to be in excellent agreement with the numerical findings. The main results and the open problems are summarized in Sec. V. Finally, the many technical details related to the stability analysis of the different regimes in the various models are confined to five Appendixes.

\section{DYNAMICAL REGIMES AND MODEL CLASSES}

As it is well known, globally coupled ensembles of identical oscillators can exhibit two highly symmetric regimes: (i) a fully synchronized state, where all the oscillators are characterized by the same phase at any time and (ii) an asynchronous regime, also called splay state, where the phases are uniformly distributed. The standard way to quantify the degree of synchronization is via the so-called Kuramoto order parameter

$$
R=N^{-1}\left|\sum_{j=1}^{N} e^{2 \pi \mathrm{i} \phi_{j}}\right|,
$$

where $N$ is the ensemble size and $\phi_{j}, j=1, \ldots, N$, is the proper phase rescaled within the unit interval. The two above-mentioned regimes correspond to: (i) $R=1$ (fully synchronous regime) and (ii) $R=0$ (asynchronous regime).

Besides such two extrema, partially synchronized states may be encountered, whose universality is less clear. Here, below we introduce two major classes: phase models (which include the Winfree model and the Kuramoto-Daido model) and pulse-coupled integrate-and-fire oscillators.

\section{A. Phase-oscillator models}

The dynamics of an autonomous limit-cycle oscillator is often described by a single equation for the phase variable. Without loss of generality this variable is introduced so that it evolves according to

$$
\dot{\phi}=v=1 / \tau,
$$

where $v(\tau)$ is the frequency (period) of the oscillation. If the given oscillator weakly interacts with its environment (weakness here means that the shape of the limit cycle is not substantially affected by the perturbation), the phase equation modifies to (see Refs. [2,22] for details and further references),

$$
\dot{\phi}=v+g Q(\phi, \psi),
$$

where $\psi$ is the phase of the forcing, $Q$ is a periodic function of both arguments, and $g$ quantifies the strength of the forcing or coupling. Without loss of generality, the constant component of $Q$ can be incorporated into frequency $v$, which then becomes $g$ dependent. In many cases $Q$ can be represented as

$$
Q(\phi, \psi)=\Gamma(\phi) Z(\psi),
$$

where $\Gamma(\phi)$ is the phase response curve (PRC) and $Z(\psi)$ is the forcing function. In globally coupled oscillators, $Z(\psi)$ can be often expressed as the sum of the contributions of the single elements, in which case, using the standard normalization $g \rightarrow g / N$, one obtains the model structure proposed long ago by Winfree to describe biological rhythms $[9,10]$,

$$
\dot{\phi}_{i}=v+g \Gamma\left(\phi_{i}\right) \frac{1}{N} \sum_{j} S\left(\phi_{j}\right) .
$$

In the weak-coupling limit $g \ll v$, the interaction, rather than being determined by the absolute phases, is determined by phase differences (see, e.g., Ref. [23]). With the help of averaging techniques, the model (5) can be indeed reduced to the so-called Kuramoto-Daido model [12]

$$
\dot{\phi}_{i}=v+\frac{g}{N} \sum_{j} G\left(\phi_{i}-\phi_{j}\right),
$$

identified by the single coupling function

$$
G(\xi)=\int_{0}^{1} \Gamma(\psi+\xi) S(\psi) d \psi .
$$

A brief derivation of this known result [21] is sketched in Appendix A. The famous Kuramoto-Sakaguchi model [11] corresponds to $G(\xi)=\sin (-\xi+\beta)$, where $\beta=$ const. The structure of the Kuramoto-Daido model can be further simplified: upon choosing a frame rotating with the common frequency $v$ one can get rid of the first term in the right-hand side. Moreover, by rescaling the time variable, one could remove the explicit dependence on the coupling constant. In order to facilitate the comparison with the other models we omit such simplifications.

\section{B. Abbott-van-Vreeswijk model}

The model consists of $N$ pulse-coupled leaky integrateand-fire (LIF) units, characterized by the scalar variables $u_{i}$, $i=1, \ldots N$, all restricted to the unit interval. In the context of neural networks, $u_{i}(t)$ is interpreted as the membrane potential; it evolves according to

$$
\dot{u}_{i}(t)=a-u_{i}+g E(t),
$$

where $a-u_{i}$ represents the velocity field that is assumed to be strictly positive (i.e., $a>1$ ), while $E(t)$ is the mean field arising from the interaction with the other oscillators and $g$ is the coupling constant. The evolution equation is complemented by a resetting rule: once the potential $u_{i}$ reaches the threshold value $u_{i}=1$, it is reset to $u_{i}=0$, the neuron fires and a spike is emitted, which contributes to the generation of the field $E$.

In a globally coupled system, the field $E$ is the linear superposition of the pulses emitted in the past by all neurons. The field dynamics can be described by an additional, linear differential equation, whose Green's function corresponds to the pulse shape [24]. In the popular model of Abbott and van Vreeswijk [20], the neuron firing at $t=t_{0}$ produces the 
so-called $\alpha$ pulse whose shape is

$$
E_{\alpha}(t)=\alpha^{2}\left(t-t_{0}\right) e^{-\alpha\left(t-t_{0}\right)} / N,
$$

where $t>t_{0}$, and the corresponding field equation reads

$$
\ddot{E}(t)+2 \alpha \dot{E}(t)+\alpha^{2} E(t)=\frac{\alpha^{2}}{N} \sum_{n \mid t_{n}<t} \delta\left(t-t_{n}\right) .
$$

From now on, the model identified by the Eqs. (8), (10) will be referred to as model $\mathrm{A}$.

\section{From the Abbott-van-Vreeswijk model to phase models}

For a proper characterization of the splay state with the help of the Kuramoto order parameter $R$, see Eq. (1), it is convenient to introduce phase $\phi \in[0,1)$ as

$$
\phi=-v \ln [1-u /(a+g v)],
$$

where $v$ is defined by the implicit formula

$$
v=-\ln ^{-1}[1-1 /(a+g v)]
$$

and $\phi(u=0)=0, \phi(u=1)=1$. As shown in Ref. [20], Eq. (8) is then transformed to

$$
\dot{\phi}_{i}=v+g \Gamma\left(\phi_{i}\right) \varepsilon,
$$

where $\varepsilon=E(t)-v$ and

$$
\Gamma(\phi)=\frac{v}{a+g v} \exp [\phi / \nu]
$$

is the PRC. In this formulation the field in the asynchronous state is $E(t)=v$ [20] and this state is characterized by $R=0$. Recall that $\phi$ is taken modulo one, unless stated otherwise.

The model structure is completed by the evolution equation for the field $\varepsilon$. Equation (10) now becomes

$$
\ddot{\varepsilon}+2 \alpha \dot{\varepsilon}+\alpha^{2} \varepsilon=\frac{\alpha^{2}}{N} \sum_{n \mid t_{n}<t}\left[\delta\left(t-t_{n}\right)-\nu\right] .
$$

Since the sum in the right-hand side can be separated into contributions from $N$ neurons, we write $\varepsilon=(1 / N) \sum_{j} S_{j}$, where $S_{j}=N \sum_{i \geqslant 1}\left(E_{\alpha}\left(t-t_{j}^{(i)}\right)-v\right)$ and $t_{j}^{(i)}$ is the time of the $i$ th spike (counted backward starting from time $t$ ) emitted by the $j$ th neuron. With this representation we recognize a Winfree-type structure (5), with a crucial difference in that $S_{j}$ cannot be expressed via the local in time value of phase, but has its own dynamics.

In the weak-coupling limit, however, the phase of each neuron increases approximately linearly in time and the spikes are equispaced [21], so that $t-t_{j}^{(i)}=t-t_{j}^{(1)}+(i-1) / v=$ $\left(\phi_{j}+i-1\right) / \nu$, where $\phi_{j}$ is the phase of the $j$ th oscillator at time $t$. As a consequence, one can turn the explicit time dependence of $S_{j}(t)$ into a phase dependence, as expected for a Winfree model. By using the definition of $E_{\alpha}$ given in Eq. (9) and resumming the corresponding series, one obtains

$$
S(\phi)=\frac{\alpha^{2}}{v} e^{-\alpha \phi / v}\left[\frac{\phi}{1-e^{-\alpha / v}}+\frac{e^{-\alpha / v}}{\left(1-e^{-\alpha / v}\right)^{2}}\right]-v .
$$

Eqs. (5), (14), (16) define model B.

Next, we introduce model C: it belongs to the KuramotoDaido class and is derived via averaging as an approximation

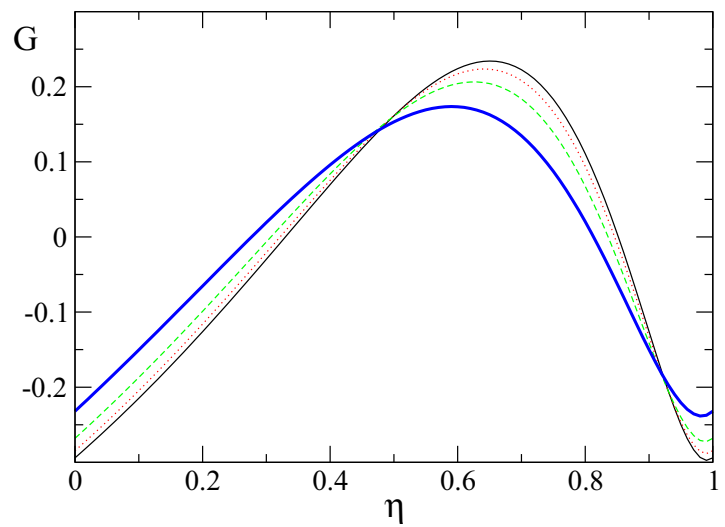

FIG. 1. (Color online) The coupling function of model $\mathrm{C}$ for different values of the coupling strength: $g=0.02$ (solid line), $g=0.05$ (dotted line), $g=0.1$ (dashed line), and $g=0.2$ (bold line). The other parameters are $a=1.3$ and $\alpha=6$. The corresponding frequency values are $v=0.6986, v=0.7747, v=0.7722$, and $v=0.8847$

of model B. For the forcing function $S$ and the PRC given by Eqs. (16), (14), Eq. (7) yields the coupling function

$$
G(\eta)=g_{1}\left(g_{2}-\eta\right) e^{\alpha \eta / v}+g_{3} e^{\eta / v}-g_{4},
$$

see Appendix B for derivation and Eq. (B1) for the $g_{n}$ coefficients. The function $G$ is plotted in Fig. 1 for some parameter values where SCPS emerges and is stable (please notice that all simulations below refer to $a=1.3$, while the other parameter values may vary). The coupling function $G$ does not reveal any special structure: it has one maximum and one minimum within the period. It can be checked, that $G(0)=G(1)$; however, $G^{\prime}(0) \neq G^{\prime}(1)$. The implications of such properties are extensively discussed in the next section.

\section{Back to pulse-coupled oscillators: a computationally efficient model}

As a corollary of the previous analysis, Winfree-type models characterized by different phase-response curves $\Gamma$ and different forcing function $S$, but identical convolution products $G$ [see Eq. (7)] are expected to be equivalent. Among them, it is instructive to consider the model with a $\delta$-like forcing function and $\Gamma(\phi)=G(\phi)$,

$$
\dot{\phi}_{i}=v+\frac{g}{N} G\left(\phi_{i}\right) \sum_{j}\left[\delta\left(\phi_{j}\right)-1\right]
$$

where we have subtracted 1 to ensure a zero average of the forcing function like in the original setup. As expected for a Winfree-type model, the argument of the $\delta$ function here is the phase. It can be transformed into a time-dependent function by substituting $\phi / \nu \rightarrow t$ into the argument of the $\delta$ function

$$
\dot{\phi}_{i}=v-g G\left(\phi_{i}\right)+\frac{g}{N v} G\left(\phi_{i}\right) \sum_{j} \delta\left(t-t_{1}\right),
$$

where $t_{1}$ is the time when any oscillator is reaching the threshold $\phi=1$. This is a standard model of $\delta$-coupled oscillators with a weakly phase-dependent velocity field. In the following we shall refer to it as to model D. 
From a computational point of view it is preferable to change variables, introducing $\theta_{i}$, according to

$$
\frac{d \phi_{i}}{d \theta_{i}}=R\left(\phi_{i}\right) \equiv \frac{v-g G\left(\phi_{i}\right)}{v_{0}},
$$

so that $\dot{\theta}_{i}=v_{0}$ [with a further adjustment of the PRC that has to be divided by $R\left(\phi_{i}\right)$ ], while the interaction terms would still be easy-to-handle $\delta$ spikes. In fact, since the time derivative between the spikes is constant, the simulation of this model does not require a differential equation solver and can be performed very efficiently. The price to pay is that $\theta$ is no longer appropriate to characterize the splay state, as the corresponding Kuramoto-order parameter would now differ from zero.

Finally, it is necessary to comment about a subtle point: since the PRC is negative for $\phi=0$, the effect of an incoming spike on the $i$ th neuron whose phase is just above zero may push it backward below zero. If one interprets $\phi$ as a true phase, this would mean that the $i$ th neuron is set below threshold and thus ready to fire again, a phenomenon that does not happen in the original formulation of the model. We should in fact interpret $\phi$ as the membrane potential $u$ in Eq. (8) and avoid the identification of $\phi<0$ with $1+\phi$.

\section{MODEL COMPARISON}

\section{A. Splay state}

The splay state and, more precisely, its stability is the first ground where the three models can be compared. The stability analysis is performed in the thermodynamic limit $N \rightarrow \infty$ by introducing the probability distribution $P(\phi, t)$ of the phases and writing the continuity equation

$$
\frac{\partial P}{\partial t}=-\frac{\partial(\dot{\phi} P)}{\partial \phi}=-\frac{\partial J}{\partial \phi},
$$

where $J$ is the corresponding current.

The three models require different approaches: for instance in model $\mathrm{A}$ it is necessary to include the field dynamics into the analysis, while model $\mathrm{C}$ does not require any perturbative expansion. In all three cases, however, in the small- $g$ limit the relevant eigenvalues can be expressed as (see Appendix $\mathrm{C}$ for a detailed account of the calculations),

$$
\mu_{n}=2 \pi \mathrm{i} n v+g \delta_{n},
$$

where

$$
\delta_{n}=\left[\frac{\alpha^{2} \nu^{2}}{a+g \nu}\right] \frac{e^{1 / v}-1}{(\alpha+2 \pi \mathrm{i} n \nu)^{2}(1+2 \pi \mathrm{i} n v)},
$$

while the corresponding eigenvectors are Fourier modes of increasing frequency. This result reveals a perfect correspondence among the three models in the weak-coupling limit.

In particular, it is interesting to notice that the splay state becomes unstable (along the direction identified by the first Fourier mode) if $\alpha$ exceeds the critical value

$$
\alpha_{c}=-1+\sqrt{1+4 \pi^{2} v^{2}} .
$$

The loss of stability in model A for $g=0.3$ was discovered in Ref. [5], where it was shown that it corresponds to the onset of SCPS (see below). Our analysis reveals that this critical

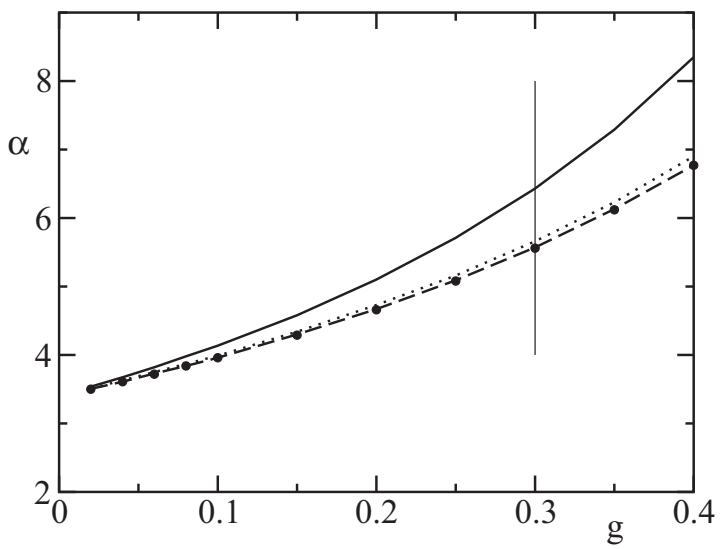

FIG. 2. Loss of splay-state stability in models A (solid curve), B (dashed curve), $\mathrm{C}$ (filled circles), and D (dotted curve). The curves for models A, B, D are obtained by numerical study of the correspondent model for $N=200$ oscillators. The curve C corresponds to the perturbative calculations, see Eq. (24). The vertical line identifies the locus of points numerically analyzed in Ref. [5].

phenomenon extends down to the weak-coupling limit and is therefore more general than initially believed.

In Fig. 2 we report the bifurcation diagram in the plane ( $g, \alpha)$, for $a=1.3$. The solid curve, obtained by simulating model A for large systems, separates the lower region, where the splay state is stable from the upper one, where SCPS is observed. The vertical straight line at $g=0.3$ corresponds to the interval of $\alpha$ values investigated by van Vreeswijk. The dashed curve corresponds to the perturbative result (24) as well as to the transition line of model $\mathrm{C}$ : it provides an excellent approximation even for relatively large $g$ values.

Quite surprisingly, numerical estimates of the transition line for model B do not reveal appreciable deviations from the perturbative prediction, suggesting that higher-order terms are almost negligible in the Winfree setup (at least up to $g \approx 1$ ). The same agreement is observed for the $\delta$-coupled oscillators in model $D$.

\section{B. Synchronous state}

While considering the synchronous regime, it is instructive to monitor not only stability but also the period $T$ of the solution as, contrary to the previous case, it is affected by the coupling strength. Let $\tau$ be the period of the uncoupled system. As follows from Eq. (12) for $g=0, \tau=-\ln (1-1 / a)$.

For model A, by making use of some general formulas derived in Ref. [25] it is found (see Appendix D) that in the weakcoupling limit the period can be written as $T=\tau+\delta T$, where

$$
\delta T=\frac{g \tau \alpha^{2}}{a} H,
$$

where

$$
H=\frac{e^{-\alpha \tau}\left(e^{\tau}-1\right)}{(\alpha-1)\left(1-e^{-\alpha \tau}\right)^{2}}-\frac{v\left(1-e^{-(\alpha-1) \tau}\right)}{(\alpha-1)^{2}\left(1-e^{-\alpha \tau}\right)} .
$$

For models $\mathrm{B}$ and $\mathrm{C}$ it is instead found that (see again Appendix D)

$$
\delta T=\frac{g \tau \alpha^{2}}{a}\left[H+\frac{v^{2}}{\alpha^{2}}\left(e^{\tau}-1\right)\right] .
$$




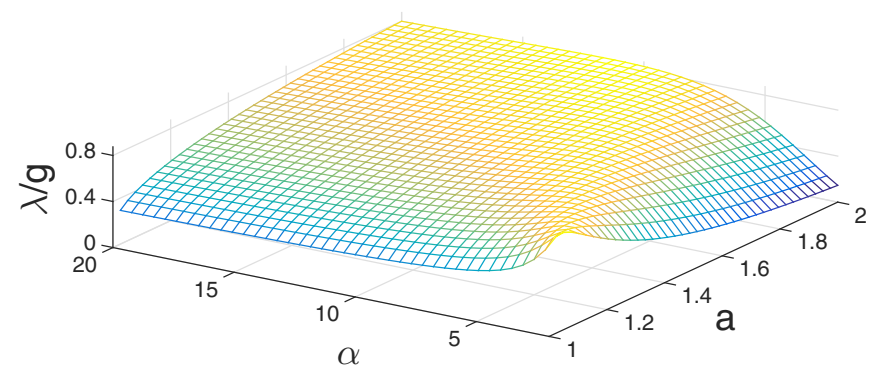

FIG. 3. (Color online) The ratio of the Lyapunov exponent and coupling strength, $\lambda / g$, versus $\alpha$ and $a$ for the synchronous solution of models A, B, see Eq. (28).

These expressions indicate that the agreement between the original LIF setup and Winfree and Kuramoto-Daido-type models is not perfect: a difference manifests itself already at the first order in $g$, i.e.,

$$
\frac{\delta T_{B, C}-\delta T_{A}}{\tau}=\frac{g}{a \tau^{2}}\left(e^{\tau}-1\right) \approx 1.2 g .
$$

Although the discrepancy is not small, it is more on a quantitative than on a qualitative level.

The stability analysis of the synchronous solution for models A and B (for $g \ll 1)$, (again performed in Appendix D) yields the Lyapunov exponent

$$
\lambda=-g \frac{\alpha^{2}}{a}\left[\frac{\alpha e^{-\alpha \tau}\left(e^{\tau}-1\right)}{(\alpha-1)\left(1-e^{-\alpha \tau}\right)^{2}}-\frac{\nu\left(1-e^{-(\alpha-1) \tau}\right)}{(\alpha-1)^{2}\left(1-e^{-\alpha \tau}\right)}\right] .
$$

For $\alpha>1$ and $g>0$ the synchronous solution turns out to be unstable, as it can be appreciated in Fig. 3.

As for the model $\mathrm{C}$, the stability of its synchronous solution is given by $\lambda=g G^{\prime}(0)$, where $G^{\prime}(0)$ is the derivative in the origin (see Appendix D): here it results in an additional difference. The point $\phi=0$ is to be identified with $\phi=1$, but the derivative of $G(\phi)$ in the two points is different: in practice, this means that the right derivative differs from the left one; Eq. (28) corresponds to the right derivative. The difference between the two derivatives is, however, somehow irrelevant, as it does not affect the sign (at least for our selection of the PRC and pulse shape).

Thus, the perturbative analysis shows that in the limit $g \ll 1, \lambda$ the Winfree and Kuramoto-Daido models are almost but not perfectly equivalent to the LIF model: the leading correction for the period of the synchronous regime differ in models $\mathrm{B}$ and $\mathrm{C}$.

\section{Partial synchronization}

Self-consistent partial synchronization has been observed only in a few setups, but the stability analysis of the splay state discussed above in this section suggests that this phenomenon might be more general than so far believed. In fact, here we show that SCPS arises in all A-D models and it can be analyzed perturbatively in the weak-coupling limit.

A way to spot SCPS is via a nonzero value of the Kuramoto order parameter $R$. In Fig. 4 it can be seen that a transition towards such a regime occurs when the inverse pulse width

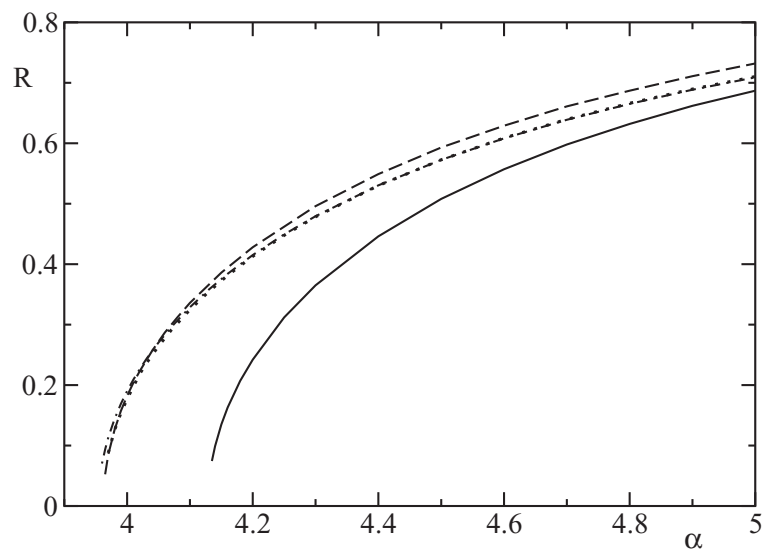

FIG. 4. Average value of the Kuramoto order parameter for $g=$ 0.1 , and $a=1.3$ : the solid, dashed, dotted, and dash-dotted lines refer to models A, B, C, and D, respectively.

$\alpha$ is increased. The curves obtained for the four models are rather close to each other, confirming an agreement that is expected from the perturbative analysis of the splay state. The more sizable deviations concern model A, suggesting that the field dynamics is not entirely negligible. Quite remarkably, the outcome of model D is also consistent (see the dash-dotted curve in Fig. 4), confirming that the effect of a smooth pulse shape can be harmlessly transferred to the PRC.

Let us now identify a signature of SCPS: a difference between the average frequency $\omega$ of the oscillators (the same for all of them) and the frequency of the mean field

$$
\Omega=\langle\dot{\Theta}\rangle, \quad \text { where } \Theta=\arg \left(N^{-1} \sum_{j} e^{\mathrm{i} \phi_{j}}\right),
$$

where $\langle\cdot\rangle$ means time average.

The results are plotted in Fig. 5 (for the same parameter values as in Fig. 4). The two frequencies are reported after

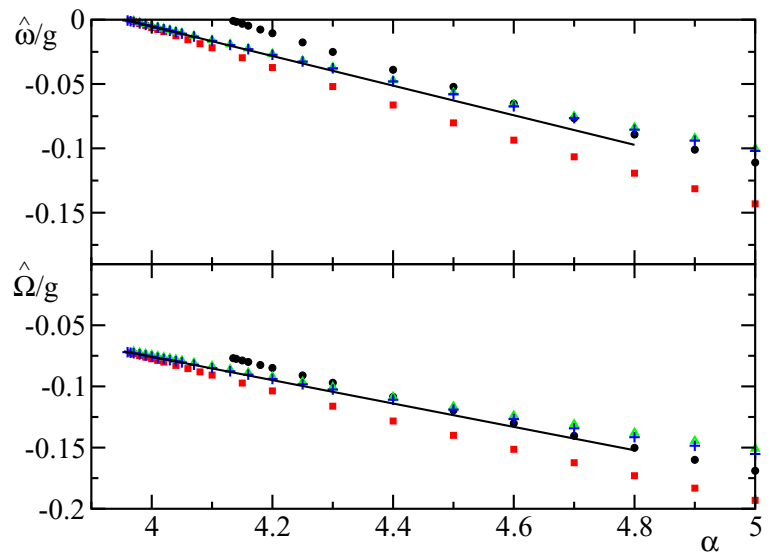

FIG. 5. (Color online) Average frequencies of the oscillators (top) and of the mean field vs $\alpha$; for $g=0.1$ and $a=1.3$. In both cases, the frequency of the splay state is subtracted, see Eq. (30), and the result is scaled with respect to the coupling strength $g$. Black circles, red squares, blue pluses, and green triangles correspond to models $\mathrm{A}, \mathrm{B}, \mathrm{C}$, and $\mathrm{D}$ respectively. The two solid curves are the outcome of the perturbative calculations carried out with model $\mathrm{C}$ (see Sec. IV). 


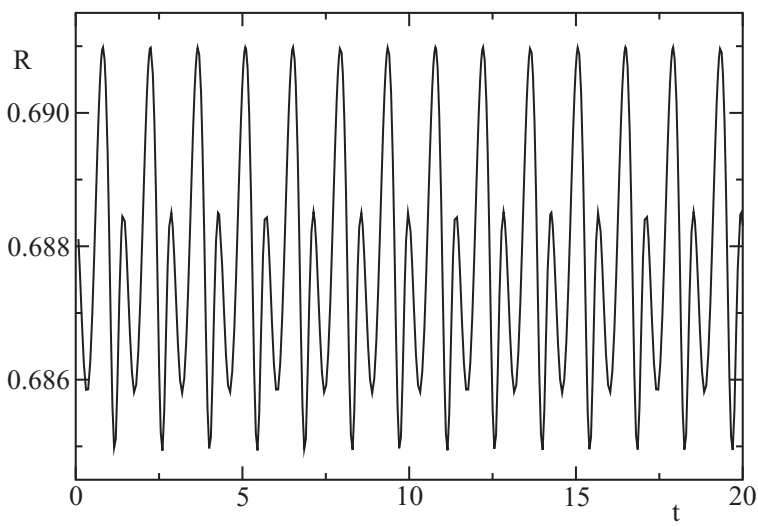

FIG. 6. Evolution of the Kuramoto order parameter in model A for $g=0.1, \alpha=5$, and $a=1.3$.

subtracting the bare frequency $v$ of the splay state to better appreciate the implication of the transition; i.e., we plot the relative frequencies

$$
\hat{\omega}=\omega-v, \quad \hat{\Omega}=\Omega-v .
$$

In Fig. 5 (top) we see that the oscillator frequency $\hat{\omega}$ vanishes at the critical $\alpha$ value below which SCPS disappears. All curves lie below zero: this means that in SCPS the oscillators are slower than in the splay state. In the bottom panel, one can see that the mean-field frequency $\hat{\Omega}$ is smaller than that of the oscillators: this is a typical signature of SCPS. It means that the oscillators move faster than their distribution. (Compare with the results for the nonlinear Kuramoto-Sakaguchi-like model in Refs. [16], where the oscillators can have any frequency relative to the mean field.) At the transition, the value of $\hat{\Omega}$ coincides with the frequency of the Hopf bifurcation. Once again, one can notice a similar kind of agreement among the three models.

Finally, we plot in Fig. 6 the time trace of the Kuramoto order parameter $R$ for the model $\mathrm{A}$ and an $\alpha$ value above threshold. There, one can see small periodic oscillations, which are still present in model B (data not shown), but completely absent in model C. As explained in the next section, this behavior is a consequence of the invariance of the evolution equations under a phase shift.

\section{PARTIAL SYNCHRONIZATION: A PERTURBATIVE APPROACH}

Within the Kuramoto-Daido setup, the forces depend on phase differences. Accordingly, there may exist nonuniform phase distributions that move rigidly in time. They can be viewed as fixed points of Eq. (21) in a suitably moving frame. The first example of such a regime was perhaps discussed in Ref. [4], where the author developed an approximate description of the LIF model in the presence of delayed pulses. Here, below we show that such states, sometimes referred to as rotating waves [26], are instances of SCPS. The representation of SCPS as a fixed point allows developing a perturbative approach and thereby deriving approximate analytical expressions to be compared with the numerics.

Let us start expressing Eq. (21) in a frame that rotates with the (yet unknown) frequency $\Omega$, by mapping $\phi \rightarrow \phi-\Omega t$, and then set $\frac{\partial P}{\partial t}=0$. By assuming that the velocity field is defined as in Eq. (6) (for $N \rightarrow \infty$ ), one obtains

$$
\frac{\partial}{\partial \phi}\left[\left(-\hat{\Omega}+g \int d \psi G(\phi-\psi) P(\psi)\right) P(\phi)\right]=0,
$$

where $\hat{\Omega}$ is an unknown quantity, to be determined selfconsistently. Upon integrating the above equation,

$$
\left[-\hat{\Omega}+g \int d \psi G(\phi-\psi) P(\psi)\right] P(\phi)=\eta=\text { const. }
$$

where the probability flux $\eta$ is also to be determined. Since phases are rescaled to the unit interval, the flux $\eta$ corresponds to the difference between the average frequency of the oscillators and that of the mean field,

$$
\eta=\hat{\omega}-\hat{\Omega}=\omega-\Omega \text {. }
$$

In general, there may be two classes of solutions of Eq. (32), characterized by $\eta=0$ and $\eta \neq 0$, respectively. In the former case, the expression in square brackets must vanish. By going in Fourier space, it can be easily seen that no such probability distribution can satisfy the condition if all Fourier components $\tilde{G}_{n} \neq 0$. On the other hand, whenever $\tilde{G}_{n}=0, \tilde{P}_{n}$ is allowed to be different from zero. Such distributions are just marginally stable and any arbitrarily small amount of noise would smooth them out. The only physically interesting solutions are those of the second class.

Determining $P(\phi)$ is not an easy task. Let us start discussing the parameter region close to the bifurcation point, where deviations from a flat distribution are small. It is convenient to rewrite Eq. (32) in Fourier space,

$$
\hat{\Omega} \sum_{n} \tilde{P}_{n} e^{-2 \pi \mathrm{i} n \phi}-g \sum_{m, n} \tilde{G}_{m} \tilde{P}_{m} \tilde{P}_{n} e^{-2 \pi \mathrm{i}(m+n) \phi}=\eta,
$$

and to decompose it into equations for the single components, obtaining

$$
\hat{\Omega} \tilde{P}_{k}-g \sum_{m} \tilde{G}_{m} \tilde{P}_{m} \tilde{P}_{k-m}=\eta \delta_{k 0} .
$$

The simulations reported in Fig. 7 suggest that higher-order harmonics are increasingly negligible upon approaching the bifurcation. Therefore, we restrict the analysis to the modes $k=1$ and $k=2$ (notice that $\tilde{P}_{0}=1$ for normalization reasons, while $\tilde{G}_{0}=0$ by definition, since the constant term of the coupling function is absorbed into the frequency). From the equation for the mode $k=0$ we obtain

$$
\tilde{G}_{1}^{r}\left|\tilde{P}_{1}\right|^{2}+\tilde{G}_{2}^{r}\left|\tilde{P}_{2}\right|^{2}=(\hat{\Omega}-\eta) / 2 g,
$$

where the superscript $r$ means that the real part is being considered. For $k=1$ and $k=2$ we find,

$$
\begin{aligned}
& \tilde{G}_{1}+\tilde{G}_{2} \tilde{P}_{2}+\tilde{G}_{1}^{*} \tilde{P}_{2}=\hat{\Omega} / g, \\
& \tilde{G}_{1} \tilde{P}_{1}^{2}+\left(\tilde{G}_{2}-\hat{\Omega} / g\right) \tilde{P}_{2}=0,
\end{aligned}
$$

where we have assumed (without loss of generality) that $\tilde{P}_{1}$ is real (the phase of the solution is arbitrary and we can set the origin as we prefer).

Let us now imagine that upon variation of the control parameter $\mu$, there exists a transition to SCPS for $\mu=\mu_{c}$. Since $\tilde{P}_{2}=0$ at the transition, from Eq. (37) it follows that, 


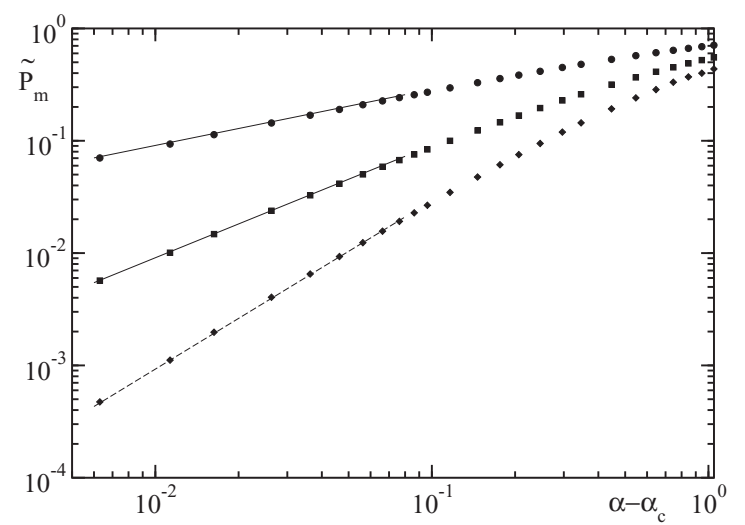

FIG. 7. First Fourier components of the phase distribution for $g=0.1$ in model $\mathrm{C}$ versus the distance from the critical point. Circles, squares, and diamonds refer to $m=1,2$, and 3, respectively. $\left|\tilde{P}_{1}\right|$ corresponds to the Kuramoto order parameter, see Eq. (1). The two solid curves (which scale with exponents $1 / 2,1$, respectively) are the outcome of the analytic calculation. The dashed curve is the outcome of a best fit with a slope $3 / 2$.

$g \tilde{G}_{1}\left(\mu_{c}\right)=\hat{\Omega}$; we call this specific value $\hat{\Omega}_{0}$. Therefore, slightly above the threshold, $g \tilde{G}_{1}=\hat{\Omega}_{0}+g \tilde{G}_{1}^{\prime} \delta \mu$ and $\hat{\Omega}=$ $\hat{\Omega}_{0}+\delta \Omega$, where the prime denotes the derivative with respect to $\mu$, while $\delta \Omega$ has to be determined. A solution of Eq. (37) is, to the leading order,

$$
\tilde{P}_{2}=\frac{\delta \Omega-g \tilde{G}_{1}^{\prime} \delta \mu}{g \tilde{G}_{2}+\hat{\Omega}_{0}},
$$

so that now Eq. (38) yields $\tilde{P}_{1}^{2}$. Next, using that $\tilde{P}_{1}$ (and thus $\left.\tilde{P}_{1}^{2}\right)$ is real, we obtain $\delta \Omega$ from the condition $\operatorname{Im}\left(\tilde{P}_{1}^{2}\right)=0$, see Appendix E for details. As a result, we find that $\delta \Omega \sim \delta \mu$, $\tilde{P}_{1}^{2} \sim \delta \mu$. A physically meaningful solution $\tilde{P}_{1} \sim \sqrt{\delta \mu}$ exists for $\delta \mu>0$, i.e., above the bifurcation point, and Eq. (38) implies that $\tilde{P}_{2}$ grows linearly and is in general complex, meaning that it is shifted with respect to the phase of $\tilde{P}_{1}$. Finally, neglecting the term proportional to $P_{2}^{2}$ in Eq. (36), we determine the last unknown, $\eta$,

$$
\hat{\Omega}-\eta=2 g \tilde{G}_{1}^{r}\left|\tilde{P}_{1}\right|^{2} .
$$

Notice that both $\tilde{\Omega}$ and the frequency difference $\hat{\Omega}-\eta$ depend linearly on the control parameter in the vicinity of the bifurcation.

These perturbative results can be compared with the numerical simulations illustrated in the previous section: $\alpha$ plays the role of the control parameter $\mu$. By computing $\tilde{P}_{1}$ and $\tilde{P}_{2}$ for $a=1.3$ and $g=0.1$ (see Appendix E), one obtains the data reported in Fig. 5. The two frequencies $\hat{\omega}$ and $\hat{\Omega}$ reveal an excellent agreement with the direct simulation of the three models. Moreover, in Fig. 7, one can see that the theoretical results (see the two upper solid lines) reproduce perfectly the behavior of the first two Fourier modes of the phase distribution.

Away from criticality, many Fourier modes come into play and a perturbative scheme is no longer effective. The distribution $P(\phi)$ can be nevertheless accurately determined by interpreting Eq. (32) as the fixed point of the recursive

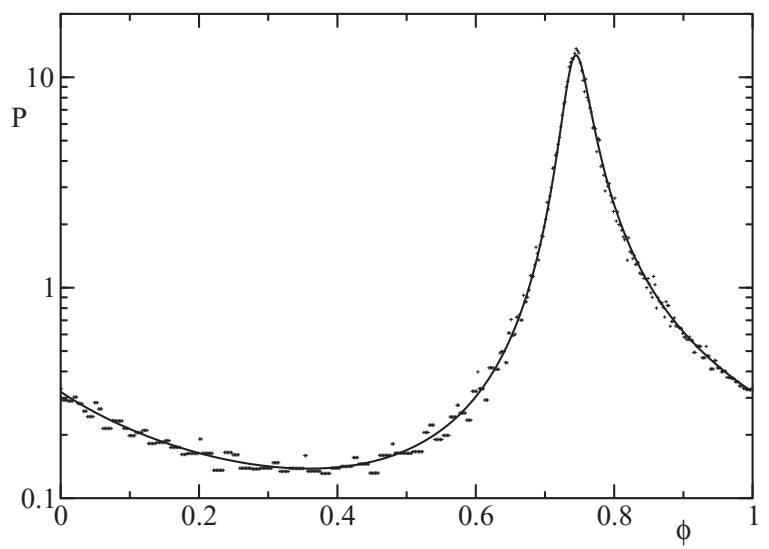

FIG. 8. Probability distribution in the partial synchronization regime for $\alpha=4.7$ for the model $\mathrm{C}$. The solid curve is the outcome of the recursive procedure discussed in the text, while the pluses refer to direct numerical simulations with $N=1000$.

relation

$$
P_{n+1}(\phi)=\frac{\eta}{g \int G(\phi-\psi) P_{n}(\psi) d \psi-\hat{\Omega}} .
$$

This equation shows that $\eta$ can be determined by imposing the normalization of the right-hand side. Numerical studies have revealed that generically the recursive procedure either converges to the flat distribution $P(\phi)=1$ or develops nonphysical negative values. We have found that upon tuning $\hat{\Omega}$, one can pass from the former to the latter regime, which are separated by a critical $\hat{\Omega}$ value for which the recursive procedure converges to a given shape with some shift. Upon changing the initial distribution, different phase shifts may be found: the correct solution is the one characterized by a zero shift (a true fixed point). Luckily, this objective can be reached by controlling a single parameter of the initial distribution: we have found that the most effective one is the width of the distribution itself. Altogether, in spite of the fact that the fixed point is an infinite-dimensional function, its shape can be determined by tuning two parameters only. The outcome of this procedure is shown in Fig. 8 for $\alpha=4.7$.

\section{SUMMARY AND OPEN PROBLEMS}

In this paper we have performed a quantitative comparison of different model classes of (phase) oscillators. A perturbative analysis of integrate-and-fire oscillators and of the corresponding Winfree and Kuramoto-Daido models reveals a substantial equivalence. The stability of the splay state is perfectly reproduced: the whole spectrum of eigenvalues coincides for all of the three models up to leading order. As for the synchronous solution, the leading correction to its frequency in the Winfree and the Kuramoto-Daido models differs from that found in the LIF model. Moreover, the Kuramoto-Daido model fails to reproduce its stability (the left stability differs from the right stability as a consequence of a nonanalyticity in the coupling function), although the difference is quantitative, but not qualitative.

The comparison has been extended to the SCPS regime, which arises from the splay state through a Hopf bifurcation. 
In this case, a mostly numerical analysis reveals again an excellent agreement among the various models. The largest deviations are observed for the LIF model, signaling that the field dynamics is not entirely negligible even in the small-coupling limit.

An important consequence of our comparative studies is the overall evidence that SCPS is not specific to integrate-andfire oscillators, but, instead, universal. In particular, it is not necessary to invoke a dependence on the order parameter, as assumed in Ref. [16].

Furthermore, the mapping of the original LIF dynamics onto a Kuramoto-Daido-type model has offered the opportunity to develop a perturbative treatment of SCPS. In fact, in such a setup, SCPS corresponds to a uniform rotation of the probability density that can be seen as a fixed point in a suitably moving frame and thereby analyzed with powerful techniques.

The actual observation of SCPS in a Kuramoto-Daido setup such as model $\mathrm{C}$ opens the question of identifying the minimal requisites for its observability. If the coupling function is composed of only one harmonics (the Kuramoto-Sakaguchi model), it is known that something similar to SCPS can be observed only in the special case of phase shift equal to $\pi / 2$, where it is, in any case, marginally stable. A subject for future research is that it is sufficient to add a second harmonic to observe a stable and robust regime of self-consistent partial synchronization.

Finally, the good correspondence between model D and the other phase models implies that restricting the study to $\delta$-coupled integrate-and-fire oscillators is not a true limitation in so far as finite pulse widths can be reduced to such a class by suitably adjusting the phase response curve. Such an equivalence has practical advantages, as the former class of models is easier to simulate.

To what extent the correspondence among the models extends to large coupling strengths is also not known: this is another point that is worth investigating in the future.

\section{ACKNOWLEDGMENT}

A.P. wishes to acknowledge the von Humboldt Foundation for the financial support, which made the collaboration possible.

\section{APPENDIX A: FROM WINFREE TO KURAMOTO-DAIDO}

In the weak-coupling limit, the dynamical changes induced by the coupling occur on long time scales compared to the period of the intrinsic oscillations and one can thereby invoke averaging techniques. With reference to the model Eq. (5), it is convenient to expand the coupling term into Fourier modes,

$$
\Gamma\left(\phi_{i}\right) S\left(\phi_{j}\right)=\sum_{n, m} \tilde{\Gamma}_{n} \tilde{S}_{m} e^{-2 \pi \mathrm{i}\left(n \phi_{i}+m \phi_{j}\right)} .
$$

By assuming that only 1:1 resonances matter and retaining the secular terms. i.e., those for which $m=-n$, one obtains

$$
\Gamma\left(\phi_{i}\right) S\left(\phi_{j}\right) \simeq \sum_{n} \tilde{\Gamma}_{n} \tilde{S}_{-n} e^{-2 \pi \mathrm{in}\left(\phi_{i}-\phi_{j}\right)}=G\left(\phi_{i}-\phi_{j}\right),
$$

so that Eq. (7) is obtained since $\tilde{S}_{-n}=\tilde{S}_{n}$.

\section{APPENDIX B: DERIVATION OF THE COUPLING FUNCTION OF MODEL C}

Using Eq. (16) together with Eqs. (7), (14) the convolution integral can be written as

$$
\begin{aligned}
G(\xi)= & \frac{\alpha^{2}}{\tilde{a}} \int e^{\frac{\psi+\xi}{v}} e^{-\frac{\alpha \psi}{v}}(A \psi+B) d \psi \\
& -\frac{\nu^{2}}{\tilde{a}} \int e^{\frac{\psi+\xi}{v}} d \psi=\frac{\alpha^{2}}{\tilde{a}} I_{1}-\frac{\nu^{2}}{\tilde{a}} I_{2},
\end{aligned}
$$

where $A=\left(1-e^{-\alpha \tau}\right)^{-1}, B=e^{-\alpha \tau}\left(1-e^{-\alpha \tau}\right)^{-2}$, and $\tilde{a}=$ $a+g \nu$. Taking into account that $\psi+\xi$ shall be understood as taken modulo one, we write

$$
\begin{aligned}
I_{1}= & e^{\xi \tau} \int_{0}^{1-\xi} e^{-(\alpha-1) \psi \tau}(A \psi+B) d \psi \\
& +e^{\xi \tau} e^{(\alpha-1) \tau} \int_{1-\xi}^{1} e^{-(\alpha-1) \psi \tau}(A \psi+B) d \psi, \\
I_{2}= & e^{\xi \tau} \int_{0}^{1-\eta} e^{\psi \tau} d \psi+e^{\xi \tau} e^{-\tau} \int_{1-\xi}^{1} e^{\psi \tau} d \psi .
\end{aligned}
$$

The further integration is straightforward; it yields Eq. (17), where the coefficients are given by the following expressions,

$$
\begin{aligned}
& g_{1}=-\frac{v \alpha^{2}\left(e^{\tau}-1\right)}{(a+g \nu)(\alpha-1)\left(e^{\alpha \tau}-1\right)}, \\
& g_{2}=\frac{1}{1-e^{-\alpha \tau}}+\frac{v}{\alpha-1}, \\
& g_{3}=\frac{v^{2} \alpha^{2}}{(a+g \nu)(\alpha-1)^{2}}, \quad g_{4}=\frac{v^{3}\left(e^{\tau}-1\right)}{a+g v} .
\end{aligned}
$$

\section{APPENDIX C: LINEAR STABILITY OF THE SPLAY STATE}

\section{Model A}

The weak-coupling limit of the splay state in this setup has been first studied in Ref. [20] and more recently extended to a broader class of pulse-coupled integrate-and-fire systems in Ref. [25]. We start from Eq. (21) with the flux

$$
J(\phi, t)=\left[v+g e^{\phi / v} \varepsilon(t)\right] P(\phi, t)
$$

and with the boundary condition $J(0, t)=J(1, t)$. The evolution equation for the field is

$$
\ddot{\varepsilon}(t)+2 \alpha \dot{\varepsilon}(t)+\alpha^{2} \varepsilon(t)=\alpha^{2}\left[J(1, t)-E_{0}\right] .
$$

The splay state corresponds to $P_{0}=1, \varepsilon=0$, and $J_{0}=$ $E_{0}=$ v

Upon introducing the perturbation $j(\phi, t)$ to the steady flux $J_{0}=v$, i.e., writing $J(\phi, t)=v+j(\phi, t)$, the evolution equations (21), (C1), (C2) can be linearized, yielding

$$
\begin{gathered}
\frac{\partial j}{\partial t}=\frac{\nu g}{\tilde{a}} e^{\phi / \nu} \frac{d \varepsilon}{d t}-\nu \frac{\partial j}{\partial \phi}, \\
\ddot{\varepsilon}(t)+2 \alpha \dot{\varepsilon}(t)+\alpha^{2} \varepsilon(t)=\alpha^{2} j(1, t) .
\end{gathered}
$$

Using the standard ansatz $j(\phi, t)=j_{f}(\phi) \exp (\mu t)$ and $\varepsilon(t)=\varepsilon_{f} \exp (\mu t)$, and imposing the boundary condition 
$j_{f}(0)=j_{f}(1)$, one obtains the eigenvalue equation

$$
\left(e^{\mu / \nu}-1\right)(\mu+\alpha)^{2}=\frac{g \alpha^{2} \mu}{\tilde{a}} \int_{0}^{1} d \phi e^{(1+\mu) \phi / v} .
$$

We now investigate the weak-coupling limit $g \ll 1$. For $g=0$, two eigenvalues are obtained by solving $(\mu+\alpha)^{2}=0$, i.e., $\mu=-\alpha$ is a double degenerate solution. Besides, the spectrum consists of an infinite set of purely imaginary eigenvalues, $\mu=2 \pi \mathrm{i} n \nu, n \neq 0$, which are most important for determination of stability. In the small $g$ limit one can assume $\mu_{n}=2 \pi \mathrm{i} n \nu+g \delta_{n}$. Upon replacing in Eq. (C5), we obtain

$$
\delta_{n}(2 \pi \mathrm{i} n \nu+\alpha)^{2}=\frac{2 \pi \mathrm{i} n \alpha^{2} \nu^{2}}{\tilde{a}} \int_{0}^{1} d \phi e^{(1 / \nu+2 \pi \mathrm{i} n) \phi} .
$$

Computing the integral, one obtains the final Eq. (23).

\section{Model B}

Here, we refer to model (5). In the thermodynamic limit, the sum over all oscillators transforms into an integral, and the expression for the probability flux takes the form

$$
J(\phi, t)=\left[v+g \Gamma(\phi) S_{P}(t)\right] P(\phi, t),
$$

where

$$
S_{P}(t)=\int_{0}^{1} d \psi S(\psi) P(\psi, t),
$$

while the boundary condition reads

$$
\left[v+g \Gamma(1) S_{P}(t)\right] P(1, t)=\left[v+g \Gamma(0) S_{P}\right] P(0, t) .
$$

At variance with the previous case, the stability can be assessed by just linearizing the above equation, without the need of including the field dynamics. The problem can be formally solved for arbitrary coupling strength.

Starting from Eqs. (C6), (C7) with the boundary condition $(\mathrm{C} 8)$, we set $P(\phi, t)=1+p(\phi, t)$, where $p(\phi, t)$ represents a perturbation around the homogeneous solution. The linearized equation writes

$$
\frac{\partial p}{\partial t}=-v \frac{\partial p}{\partial \phi}-g \Gamma^{\prime}(\phi) S_{p},
$$

where the prime denotes derivation with respect to $\phi$ and $S_{p}$ is defined analogously to $S_{P}$, see Eq. (C7); notice also that $S_{P}=0$ in the splay state. The boundary condition becomes

$$
v[p(1, t)-p(0, t)]=-g S_{p} \Delta \Gamma,
$$

where $\Delta \Gamma=\Gamma(1)-\Gamma(0)$.

Next, we introduce the usual ansatz, $p(\phi, t)=\rho(\phi) e^{\mu t}$, obtaining

$$
v \frac{d \rho}{d \phi}=-\mu \rho-g \Gamma^{\prime}(\phi) S_{\rho},
$$

where $S_{\rho}$ is defined analogously to $S_{P}$, see Eq. (C7). By assuming that $\rho(\phi)=\rho_{0}(\phi) \exp (-\mu \phi / \nu)$, we find that

$$
\rho_{0}(\phi)=-\frac{g}{v} S_{\rho} I_{\mu}(\phi)+C,
$$

where

$$
I_{\mu}(\phi)=\int_{0}^{\phi} d \xi \Gamma^{\prime}(\xi) e^{\mu \xi / \nu}
$$

The integration constant can be determined from the boundary condition

$$
C=\frac{g}{v} \frac{e^{-\mu / v} I_{\mu}(1)-\Delta \Gamma}{e^{-\mu / v}-1} S_{\rho}
$$

As a result,

$$
\rho(\phi)=\frac{g}{\nu} e^{-\mu \phi / \nu}\left[\frac{e^{-\mu / \nu} I_{\mu}(1)-\Delta \Gamma}{e^{-\mu / \nu}-1}-I_{\mu}(\phi)\right] S_{\rho} .
$$

The eigenvalue equation is finally obtained by multiplying $\rho(\phi)$ by $S(\phi)$ and integrating over $\phi$ to obtain $S_{\rho}$ :

$$
g \frac{e^{-\mu / v} I_{\mu}(1)-\Delta \Gamma}{e^{-\mu / v}-1}\left\langle e^{-\mu \phi / v}\right\rangle_{S}-g\left\langle I_{\mu}(\phi) e^{-\mu \phi / v}\right\rangle_{S}=v,
$$

where $\langle\cdot\rangle_{S}$ denotes the integral over the dummy variable $\phi$ after having been multiplied by $S(\phi)$.

In the weak-coupling limit, the second addendum in the lefthand side of the above equation can be neglected, while the first one can be properly handled by assuming $\mu_{n}=2 \pi \mathrm{i} n v+g \delta_{n}$ in the numerator (and $\mu_{n}=2 \pi$ inv everywhere else). As a result, the eigenvalue equation simplifies to

$$
\delta_{n}=-\left[\tilde{\Gamma}_{n}^{\prime}-\Delta \Gamma\right] \tilde{S}_{n}^{*},
$$

since Eq. (C12) reduces to the Fourier transform of $\Gamma^{\prime}$, while $\left\langle e^{-\mu \phi / \nu}\right\rangle_{S}$ reduces to the conjugate of the transform of $S$. From Eq. (14), it follows that

$$
\tilde{\Gamma}_{n}=\int_{0}^{1} d \phi \Gamma(\phi) e^{2 \pi \mathrm{in} \phi}=\frac{v}{\tilde{a}} \frac{e^{1 / v}-1}{1 / v+2 \pi \mathrm{i} n},
$$

and, accordingly,

$$
\tilde{\Gamma}_{n}^{\prime}=\frac{1}{\tilde{a}} \frac{e^{1 / v}-1}{1 / v+2 \pi \mathrm{i} n}
$$

so that

$$
\tilde{\Gamma}_{n}^{\prime}-\Delta \Gamma=-\frac{e^{1 / v}-1}{\tilde{a}} \frac{2 \pi \mathrm{i} n v}{1 / v+2 \pi \mathrm{i} n} .
$$

By further noticing that

$$
\tilde{S}_{n}=\int_{0}^{1} d \phi S(\phi) e^{2 \pi \mathrm{in} \phi}=\frac{\alpha^{2} v}{(\alpha-2 \pi \mathrm{i} n v)^{2}} .
$$

we finally obtain Eq. (23).

\section{Model C}

In the thermodynamic limit, Eq. (6) can be written as

$$
\dot{\phi}=v+g \int d \psi G(\phi-\psi) P(\psi),
$$

or, using the Fourier representation, as

$$
\dot{\phi}=v+g \sum_{n} \tilde{G}_{n} \tilde{P}_{n} e^{-2 \pi \mathrm{i} n \phi} .
$$

Accordingly, the continuity equation becomes

$$
\frac{\partial P}{\partial t}=-\frac{\partial}{\partial \phi}\left[\left(v+g \sum_{n} \tilde{G}_{n} \tilde{P}_{n} e^{-2 \pi \mathrm{i} n \phi}\right) P(\phi, t)\right] .
$$


We now linearize Eq. (C23) around the splay solution $P_{0}(\phi)=1$, by assuming $P(\phi, t)=1+p(\phi, t)$. Since the mode amplitudes of the equilibrium solution for $n \neq 0$ are all equal to zero,

$$
\frac{\partial p}{\partial t}=-v \frac{\partial p}{\partial \phi}+2 \pi \mathrm{i} g \sum_{n \neq 0} n \tilde{G}_{n} \tilde{p}_{n} e^{-2 \pi \mathrm{i} n \phi} .
$$

At variance with the previous setups, one can easily solve the continuity equation by just going in Fourier space, as this change of variables diagonalizes the evolution equation for any parameter value

$$
\frac{d \tilde{p}_{n}}{d t}=2 \pi \mathrm{i} n\left[v+g \tilde{G}_{n}\right] \tilde{p}_{n}
$$

By recalling that $\mu_{n} \equiv 2 \pi \mathrm{i} n \nu+g \delta_{n}$,

$$
\delta_{n}=2 \pi \mathrm{i} n \tilde{G}_{n}=2 \pi \mathrm{i} n \tilde{\Gamma}_{n} \tilde{S}_{n}^{*},
$$

which, in the case of the LIF model, coincides with Eq. (23).

\section{APPENDIX D: LINEAR STABILITY OF THE SYNCHRONOUS STATE}

\section{Model A}

From Eq. (52) in Ref. [25], the period $T$ for the ensemble of LIF oscillators with $\alpha$ pulses is determined by the implicit condition

$$
a\left(1-e^{-T}\right)+g\left[\frac{e^{-T}-e^{-\alpha T}}{\alpha-1}(V+Q)-T e^{-\alpha T} Q\right]=1,
$$

where

$$
Q=\frac{\alpha^{2} /(\alpha-1)}{1-e^{-\alpha T}}, \quad V=\frac{\alpha^{2} T e^{-\alpha T}}{\left(1-e^{-\alpha T}\right)^{2}} .
$$

For $g=0$, the period is equal to $\tau=-\ln (1-1 / a) \equiv 1 / \nu$, cf. Eq. (12); let us denote with $Q_{0}, V_{0}$ the corresponding values of $Q$ and $V$. In the small $g$ limit, we can assume $T=\tau+\delta T$, where $\delta T$ is small, and expand the first term in Eq. (D1) (the second term is already of order $g$ ), obtaining

$$
\delta T=-\frac{g}{a}\left[\frac{1-e^{-(\alpha-1) \tau}}{\alpha-1}\left(V_{0}+Q_{0}\right)-\tau e^{-(\alpha-1) \tau} Q_{0}\right] .
$$

By replacing the expressions for $V_{0}$ and $Q_{0}$, we finally obtain Eqs. (25), (26). [25],

The stability of the limit cycle is determined by the exponent

$$
\lambda=\frac{1}{T} \ln \frac{a+g V}{a-1+g V}-1 .
$$

By now expanding for $g \ll 1$, we obtain, up to the first order,

$$
\lambda=-\frac{\delta T}{\tau}-\frac{g V_{0}}{\tau a(a-1)} .
$$

With the help of Eq. (25) and recalling that $e^{\tau}-1=1 /(a-1)$, one obtains the final expression for the Lyapunov exponent that is reported in Eq. (28).

\section{Model B}

Here, we determine the period and determine the stability of the fully synchronous solution of the model (5). In the weak-coupling limit, the period can be estimated through a perturbative calculation, by setting $\phi=v t+\beta(t)$ in Eq. (5) and retaining the leading order,

$$
\dot{\beta}=g \Gamma(v t) S(v t) \text {. }
$$

The period $T$ can be then obtained by solving the above equation and imposing

$$
v T+\beta(\tau)=1,
$$

so that

$$
\delta T=-\tau \beta(\tau) .
$$

$\beta(\tau)$ can be determined by integrating Eq. (D6) that can be written as,

$$
\dot{\beta}=\frac{g \alpha^{2}}{a}\left[\frac{v t e^{-(\alpha-1) t}}{1-e^{-\alpha / v}}+\frac{e^{-\alpha / v} e^{-(\alpha-1) t}}{\left(1-e^{-\alpha / v}\right)^{2}}-\frac{v^{2}}{\alpha^{2}} e^{t}\right],
$$

where we have used that $\tilde{a}=a$ in the weak-coupling limit. By replacing the integral of this equation into Eq. (D8), one obtains the expression reported in Eq. (27).

As for the stability, the tangent space evolution writes

$$
\frac{d \delta \phi_{i}}{d t}=g \Gamma^{\prime}\left(\phi_{i}\right)\langle S\rangle \delta \phi_{i}+g \Gamma\left(\phi_{i}\right) \frac{1}{N} \sum S^{\prime}\left(\phi_{j}\right) \delta \phi_{j} .
$$

If all the oscillators are synchronized, we can drop the index dependence in the phase-space dynamics,

$$
\frac{d \delta \phi_{i}}{d t}=g \Gamma^{\prime}(\phi) S(\phi) \delta \phi_{i}+g \Gamma(\phi) \frac{S^{\prime}(\phi)}{N} \sum_{j} \delta \phi_{j} .
$$

The stability can be assessed by introducing the variables $\theta_{i}=$ $\delta \phi_{i}-\delta \phi_{1}$ with $i \geqslant 2$ [27] (the sum of all $\delta \phi_{i}$ gives a missing equation, which is known to yield the zero exponent and we thereby avoid considering it),

$$
\dot{\theta}_{i}=g \Gamma^{\prime}(\phi) S(\phi) \theta_{i} .
$$

Since $\Gamma(\phi)$ is discontinuous for $\phi=1$, its derivative has a $\delta$ contribution that has to be properly included in the computation of the Floquet exponent. The final result is

$$
\lambda=\frac{1}{T} \ln \frac{\theta(T)}{\theta(0)}+\frac{1}{T} \ln \frac{v+g \Gamma(0) S(0)}{v+g \Gamma(1) S(1)} .
$$

In the limit $g \ll 1$, taking into account that $S(0)=S(1)$ the above equation reduces to

$$
\lambda=\frac{1}{\tau} \ln \frac{\theta(\tau)}{\theta(0)}+G[\Gamma(0)-\Gamma(1)] S(0) .
$$

One can then determine $\theta(\tau)$ by integrating Eq. (D12) with the same philosophy as for Eq. (D6). As a result the same expression as (28) is obtained for $\lambda$.

\section{Model C}

The determination of the period is pretty straightforward: it can be obtained by setting the argument of the interaction function $G$ equal to zero

$$
\frac{1}{T}=v+g G(0),
$$

so that, for the LIF oscillators,

$$
\delta T=-g G(0) \tau^{2}=g\left(g_{1} g_{2}+g_{3}-g_{4}\right) \tau^{2} .
$$


Upon replacing the expressions for $g_{1}, g_{2}, g_{3}$, and $g_{4}$ reported in Appendix B, one can verify that the above equation coincides with Eq. (27).

Next, we linearize the equations of motion, obtaining

$$
\frac{d \delta \phi_{i}}{d t}=g G^{\prime}(0) \delta \phi_{i}-\frac{g}{N} G^{\prime}(0) \sum_{j}-\delta \phi_{j} .
$$

The stability can be determined by again introducing the variables $\theta_{i}=\delta \phi_{i}-\delta \phi_{1}$, which satisfy the following equation

$$
\dot{\theta}_{i}=g G^{\prime}(0) \theta_{i},
$$

so that the stability is controlled by the sign of $G^{\prime}(0)$.

\section{APPENDIX E: COMPUTATION OF THE FIRST FOURIER MODE OF THE PROBABILITY DISTRIBUTION IN SCPS STATE}

Substituting Eq. (38) into Eq. (39), we obtain

$$
\tilde{P}_{1}^{2}=\left(\frac{\hat{\Omega}_{0}-g \tilde{G}_{2}}{\hat{\Omega}_{0}+g \tilde{G}_{2}}\right)\left(\frac{\delta \Omega-g \tilde{G}_{1}^{\prime} \delta \mu}{\hat{\Omega}_{0}}\right) .
$$

Condition $\operatorname{Im}\left(\tilde{P}_{1}^{2}\right)=0$ yields

$$
\delta \Omega=\left[\left(\tilde{G}_{1}^{\prime}\right)^{r}+\left(\tilde{G}_{1}^{\prime}\right)^{i} \frac{g^{2}\left|\tilde{G}_{2}\right|^{2}-\hat{\Omega}_{0}^{2}}{2 g \hat{\Omega}_{0} \tilde{G}_{2}^{i}}\right] \delta \mu=M \delta \mu .
$$

As a result, from Eq. (E1), it follows that $\tilde{P}_{1}^{2}$ is proportional to $\delta \mu$,

$$
\tilde{P}_{1}^{2}=\left(\frac{\hat{\Omega}_{0}-g \tilde{G}_{2}}{\hat{\Omega}_{0}+g \tilde{G}_{2}}\right)\left(\frac{M-g \tilde{G}_{1}^{\prime}}{\hat{\Omega}_{0}}\right) \delta \mu .
$$

To complete the computation we have to find $\tilde{G}_{2}, \tilde{G}_{1}^{\prime}$ at the bifurcation point. With the reference to the Abbot-vanVreeswijk model, the coupling function is given by Eq. (17) and the role of the order parameter is played by the inverse pulse width $\alpha$; the bifurcation value $\alpha_{c}$ is given by Eq. (24). Computing the first two Fourier modes of $G$, we find:

$$
\tilde{G}_{1,2}=C \frac{A_{1,2}-\mathrm{i} B_{1,2}}{D_{1,2}},
$$

where

$$
C=\frac{\alpha_{c}^{2} v^{3}}{a+g v}\left(e^{1 / v}-1\right)
$$

and

$$
\begin{aligned}
& A_{n}=\alpha_{c}^{2}-(2 \pi n v)^{2}\left(1+2 \alpha_{c}\right), \\
& B_{n}=2 \pi n v\left[\alpha_{c}^{2}+2 \alpha_{c}-(2 \pi n v)^{2}\right], \\
& D_{n}=\left[\alpha_{c}^{2}+(2 \pi n v)^{2}\right]^{2}\left[1+(2 \pi n v)^{2}\right] .
\end{aligned}
$$

Finally,

$$
\begin{aligned}
& \left(\tilde{G}_{1}^{\prime}\right)^{r}=\frac{2 C}{D_{1}}\left(\frac{A_{1}}{\alpha_{c}}-\frac{2 \alpha_{c} A_{1}}{4 \pi^{2} v^{2}+\alpha_{c}^{2}}+\alpha_{c}-4 \pi^{2} v^{2}\right), \\
& \left(\tilde{G}_{1}^{\prime}\right)^{i}=-\frac{4 C \pi v\left(1+\alpha_{c}\right)}{D_{1}} .
\end{aligned}
$$

[1] Y. Kuramoto, in International Symposium on Mathematical Problems in Theoretical Physics, edited by H. Araki, Lecture Notes in Physics Vol. 39 (Springer, New York, 1975), p. 420.

[2] Y. Kuramoto, Chemical Oscillations, Waves and Turbulence (Springer, Berlin, 1984).

[3] J. A. Acebron, L. L. Bonilla, C. J. P. Vicente, F. Ritort, and R. Spigler, Rev. Mod. Phys. 77, 137 (2005).

[4] Y. Kuramoto, Physica D: Nonlinear Phenomena 50, 15 (1991).

[5] C. van Vreeswijk, Phys. Rev. E 54, 5522 (1996).

[6] P. Mohanty and A. Politi, J. Phys. A: Math. Gen. 39, L415 (2006).

[7] Y. Kuramoto and D. Battogtokh, Nonlin. Phenom. Complex. Syst. 5, 380 (2002).

[8] D. M. Abrams and S. H. Strogatz, Phys. Rev. Lett. 93, 174102 (2004).

[9] A. T. Winfree, J. Theor. Biol. 16, 15 (1967).

[10] A. T. Winfree, The Geometry of Biological Time (Springer, Berlin, 1980).

[11] H. Sakaguchi and Y. Kuramoto, Prog. Theor. Phys. 76, 576 (1986).

[12] H. Daido, Prog. Theor. Phys. 88, 1213 (1992); 89, 929 (1993); Physica D 91, 24 (1996).

[13] C. S. Peskin, Mathematical Aspects of Heart Physiology (Courant Institute of Mathematical Sciences, New York, 1975).

[14] R. Mirollo and S. Strogatz, SIAM J. Appl. Math. 50, 1645 (1990).
[15] L. Lapicque, J. Physiol. Pathol. Gen. 9, 620 (1907).

[16] M. Rosenblum and A. Pikovsky, Phys. Rev. Lett. 98, 064101 (2007); A. Pikovsky and M. Rosenblum, Physica D 238, 27 (2009).

[17] S. Luccioli and A. Politi, Phys. Rev. Lett. 105, 158104 (2010).

[18] S. Watanabe and S. H. Strogatz, Phys. Rev. Lett. 70, 2391 (1993); Physica D: Nonlinear Phenomena 74, 197 (1994).

[19] A. Pikovsky and M. Rosenblum, Physica D 240, 872 (2011).

[20] L. F. Abbott and C. van Vreeswijk, Phys. Rev. E 48, 1483 (1993).

[21] D. Golomb, D. Hansel, and G. Mato, in Neuro-informatics and Neural Modeling, Handbook of Biological Physics Vol. 4, edited by F. Moss and S. Gielen (Elsevier, Amsterdam, 2001), pp. 887-968.

[22] A. Pikovsky, M. Rosenblum, and J. Kurths, Synchronization. A Universal Concept in Nonlinear Sciences. (Cambridge University Press, Cambridge, 2001).

[23] G. B. Ermentrout and N. Kopell, J. Math. Biol. 29, 195 (1991).

[24] S. Olmi, A. Politi, and A. Torcini, J. Math. Neurosci. 2, 12 (2012).

[25] S. Olmi, A. Politi, and A. Torcini, Europhys. Lett. 92, 60007 (2010).

[26] D. Hansel, G. Mato, and C. Meunier, Neural Comput. 7, 307 (1995).

[27] S. H. Strogatz, Nonlinear Dynamics and Chaos: With Applications to Physics, Biology, Chemistry, and Engineering (Addison-Wesley, Reading, Massachusetts, 1994). 